\title{
Strategies for Enhancing Vaccine-Induced CTL Antitumor Immune Responses
}

\author{
Xin Yong, ${ }^{1}$ Yü-Feng Xiao, ${ }^{1}$ Gang Luo, ${ }^{1}$ Bin He, ${ }^{1}$ Mu-Han Lü, ${ }^{1}$ Chang-Jiang Hu, ${ }^{1}$ \\ Hong Guo, ${ }^{1}$ and Shi-Ming Yang ${ }^{1,2,3}$ \\ ${ }^{1}$ Department of Gastroenterology, Xinqiao Hospital, Third Military Medical University, Chongqing 400037, China \\ ${ }^{2}$ Biomedical Analysis Center, Third Military Medical University, Chongqing 400038, China \\ ${ }^{3}$ Chongqing Key Laboratory for Diseases Proteomics, Southwest Hospital, Third Military Medical University, Chongqing 400038, China
}

Correspondence should be addressed to Shi-Ming Yang, shimingyang@yahoo.com

Received 8 August 2012; Accepted 13 September 2012

Academic Editor: Jeffrey A. Frelinger

Copyright ( $) 2012$ Xin Yong et al. This is an open access article distributed under the Creative Commons Attribution License, which permits unrestricted use, distribution, and reproduction in any medium, provided the original work is properly cited.

\begin{abstract}
Vaccine-induced cytotoxic T lymphocytes (CTLs) play a critical role in adaptive immunity against cancers. An important goal of current vaccine research is to induce durable and long-lasting functional CTLs that can mediate cytotoxic effects on tumor cells. To attain this goal, there are four distinct steps that must be achieved. To initiate a vaccine-induced CTL antitumor immune response, dendritic cells (DCs) must capture antigens derived from exogenous tumor vaccines in vivo or autologous DCs directly loaded in vitro with tumor antigens must be injected. Next, tumor-antigen-loaded DCs must activate CTLs in lymphoid organs. Subsequently, activated CTLs must enter the tumor microenvironment to perform their functions, at which point a variety of negative regulatory signals suppress the immune response. Finally, CTL-mediated cytotoxic effects must overcome the tolerance induced by tumor cells. Each step is a complex process that may be impeded in many ways. However, if these steps happen under appropriate regulation, the vaccine-induced CTL antitumor immune response will be more successful. For this reason, we should gain a better understanding of the basic mechanisms that govern the immune response. This paper, based on the steps necessary to induce an immune response, discusses current strategies for enhancing vaccine-induced CTL antitumor immune responses.
\end{abstract}

\section{Introduction}

Cancer is the leading cause of death in economically developed countries and the second leading cause of death in developing countries [1]. Advancements have been made in traditional treatment modalities that have been used for decades, namely, surgery, radiotherapy, and chemotherapy. In addition, with the value of early diagnosis in cancer therapy recognized, the technology of early diagnosis is also advancing. Although these treatment modalities play an important role, the results are not entirely adequate, especially for advanced cancers. Cancer is still a major public health problem worldwide, and new treatment modalities and strategies are still needed to optimize patient outcomes.

Cancer immunotherapy, which can be generally classified as passive or active, has always been an attractive and potentially efficient treatment for cancer patients [2]. Passive immunotherapy, consisting of infusion of donor T lymphocytes and transfer of anticancer monoclonal antibodies, has been proven to be an effective treatment for a variety of cancers $[3,4]$, and continued advances in T-cell engineering and antibody should further enhance their clinical impact. However, vaccines, which represent active immunotherapy, are based on the manipulation of the host immune system to fight cancer and provide a path to obtain long-lasting responses in cancer patients [5]. As one of the major players in active immunity, cytotoxic T lymphocytes (CTLs) play a critical role in immunity against cancers. The goal of vaccines is to induce durable and long-lasting functional CTLs. A variety of vaccine strategies have been designed to meet this goal, and recent phase II/III clinical trials using these vaccines have achieved promising results [6].

Cancer vaccines enhance the antitumor immune response by providing the early signals of activity; dendritic 
cells (DCs) play an important role in this immune response activation, which involves a number of complex processes. First, DCs must capture tumor antigens, process the captured antigen for presentation on major histocompatibility complex (MHC) molecules (either class I or class II), and then migrate to draining lymph nodes. If capture and processing is accompanied by a suitable activation signal, DCs will enhance the activation of the immune response. If not, DCs will instead induce tolerance [7]. Second, in lymphoid organs, tumor-antigen-loaded DCs are capable of triggering protective T-cell responses, especially CTL responses [8]. In this process, DCs require a maturation signal (i.e., a stimulatory adjuvant) in order to elicit the desired CTLs [9, 10]. Without a maturation signal, DCs present antigens in a stable state, which promotes tolerance by inducing regulatory $\mathrm{T}$ cell $\left(\mathrm{T}_{\text {reg }}\right)$ production and thereby thwarting an antitumor response [11-13]. In addition, the ability of DCs to promote a CTL response also depends on the interaction of a positive T-cell costimulatory molecule (i.e., CD28, OX40) with DC surface receptors (i.e., CD80/CD86, OX40L); the interaction of the negative T-cell costimulatory molecule (i.e., CTLA-4, LAG-3) with the DC surface receptors can limit the activity of CTLs by promoting $\mathrm{T}_{\text {reg }}$ formation. For these reasons, the appropriate utilization and regulation of DCs correlate with the success or failure of vaccine design. All in all, increasing immune activation by DCs is a critical step for improving CTL antitumor immune responses (Figure 1).

Targeted therapy is a typical representative of selective, mechanism-based therapy and has become a new treatment option. Based on the molecular mechanisms of CTL activation, targeted drugs can not only promote the capture of tumor antigens by DCs can also enhance the expression of costimulatory molecules such as CD40, CD80, and CD86 on the DC surface. Moreover, targeted drugs block important inhibitory signals for activated T-cells, thereby maintaining $\mathrm{T}$ cell activation and potentiating tumor destruction [14]. In general, through regulation of DCs and activated T cells, targeted drugs effectively boost CTL activation [15-17]. Furthermore, activated CTLs must enter the tumor microenvironment to perform their functions, at which point a variety of negative regulatory signals suppress the immune response; CTLs must overcome the tolerance induced by tumor cells in order to mediate their cytotoxic effects. Targeted drugs aim to inhibit these negative factors [18-20]. Therefore, the effects of drugs that target the processes that generate CTL antitumor immune responses can be complementary and synergistic with cancer vaccines. Combinatorial therapy provides a new treatment modality that enhances the vaccine-induced CTL antitumor immune response. However, greater focus on the appropriate dosage, sequencing, and timing of the targeted drugs is needed, as it will probably be crucial to the success of combinatorial strategies. Another key issue for combinatorial therapies is the toxicity of these regimens.

In general, the ultimate goal of enhancing vaccineinduced CTL antitumor immune responses is to improve therapeutic efficacy. Encouraged by positive preclinical and clinical data [21-25], the research into the appropriate utilization and regulation of DCs in vaccine design continues, and the study of the combination of vaccines and targeted drugs aims to solve the above-mentioned problems.

\section{Facilitating the Capture of Tumor Antigens by Dendritic Cells}

DCs, as the most efficient antigen-presenting cells (APCs), have been increasingly used in various strategies of vaccine design to enhance their unique ability to activate antitumor CTLs. These strategies facilitate the capture of tumor antigens by DCs, which helps to stimulate the activation of CTLs in the next step.

In the past few decades, vaccine design strategy has included several antigen-loading techniques to activate DCs, such as (i) pulsing DCs with peptides or proteins, (ii) transfecting DCs with DNA or RNA, (iii) loading DCs with tumor-cell lysates or tumor cells, and (iv) infecting DCs with bacterial, viral, or yeast vectors [26]. (Figure 1(a)) In contrast, vaccines that do not utilize DCs tend to depend on the immunogenicity of the vaccines itself, which are not enough to elicit a stronger CTL response $[27,28]$. DCs transfected with the human telomerase reverse transcriptase (hTERT)-HIL18 gene were capable of eliciting a stronger hTERT-specific CTL response in vitro than those stimulated with the hTERT construct only [29]. This strategy of vaccine design utilizes DC activation in vitro in order to avoid the complex regulation of DC exposure to antigen in vivo.

On antigen encounter in vivo, DCs require a suitable activation and maturation signal to promote immunity (enhanced capture, processing and presentation of tumor antigen-derived peptides) rather than tolerance $[7,30]$. These signals include endogenous tumor cell lysates (e.g., high mobility group proteins or ATP) and exogenous molecules (e.g., Toll-like receptor (TLR) ligands or antibodies). Some exogenous molecules have been used in preclinical and clinical. Like TLR ligands (which will be discussed later in this paper), monoclonal antibodies can been used to facilitate the capture of tumor antigens by DCs. Trastuzumab and cetuximab are clinically efficacious monoclonal antibodies that are directed toward the tumor-associated receptor tyrosine kinases HER2 and EGFR, respectively. These antibodies not only downregulate oncogenic signaling, but also enhance the induction of tumor-specific CTLs [31, 32]. These antibodies coat tumor-associated receptors on tumor cells and bind Fc receptors expressed on DCs simultaneously to form immune complexes that contribute to the capture of tumor antigens by DCs. Further, Fc receptor-mediated opsonization enhances the expression of costimulatory molecules (i.e., CD40, CD80, and CD86) on the DC surface, boosting T-cell activation [33]. Therefore, direct inoculation of patients with vaccines that activate DCs in vivo requires combining this targeted therapy in order to promote a desired immunogenic phenotype. Wolpoe et al. confirmed that combining a cancer cell vaccine and HER2 antibodies resulted in significantly longer survival rates than either therapy alone in a transgenic mouse model of breast cancer [32]. This may be due to the fact that the combination therapy increased the capture of the cancer vaccine by DCs, enhanced the expression of costimulatory molecules 


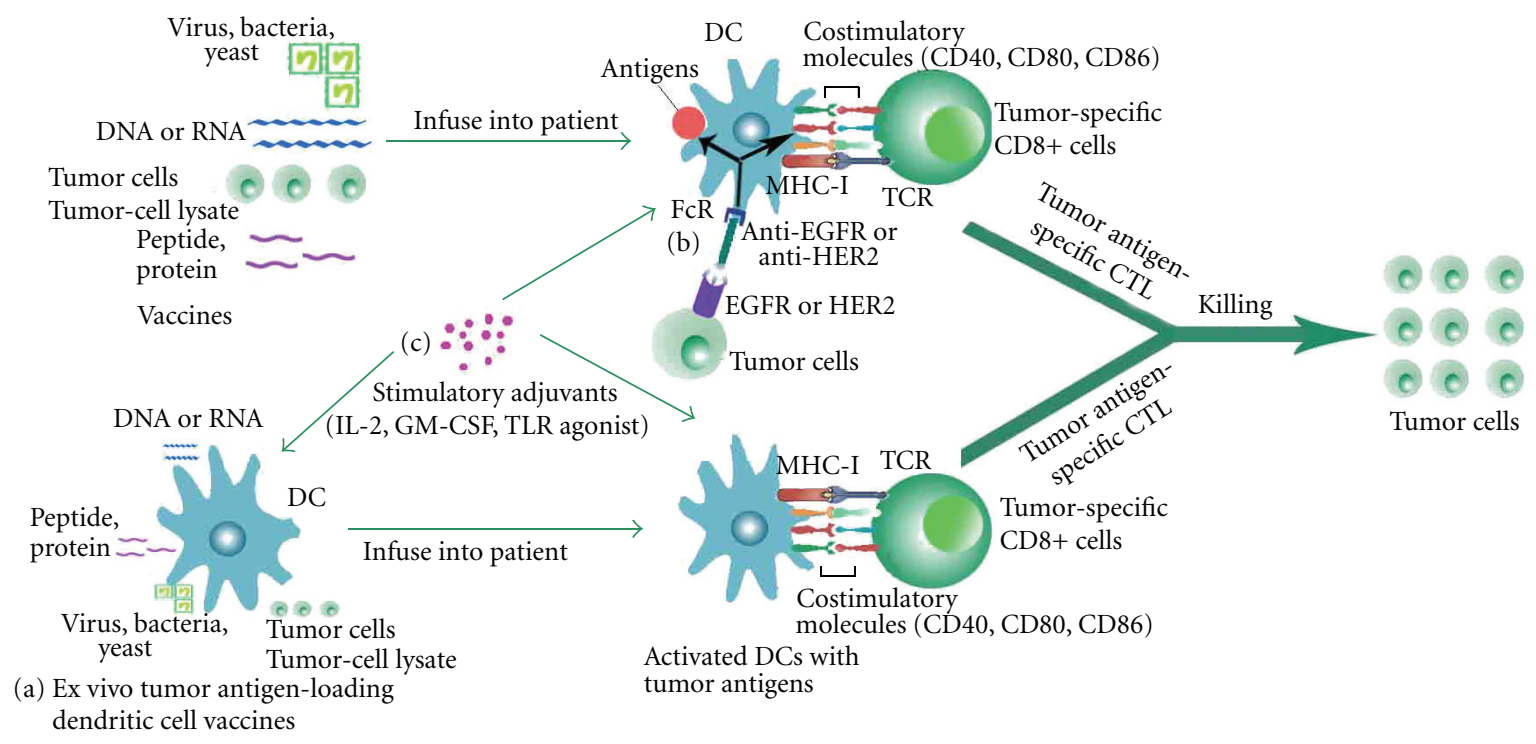

FIGURE 1: Appropriate utilization and regulation of DCs in vaccine design induce a much more potent CTL antitumor immune response. (a) Tumor antigen-loading techniques activate DCs ex vivo. (b) Targeted drugs facilitate the capture of tumor antigens by DCs and the expression of costimulatory molecules and MHC-II in vivo. (c) Stimulatory adjuvants induce maturation of DCs and enhance the activation of CTLs.

(i.e., CD40, CD80, and CD86) on the DCs, and elicited greater tumor-specific T-cell responses [34]. Recently, a Phase I/II clinical trial of trastuzumab combined with a HER2 peptide vaccine showed that $69 \%$ of patients developed CTL immune responses to tumor cells that expressed HER2 [35]. Similarly, the EGFR antibody cetuximab also enhances DC-mediated phagocytosis, increases the expression of the MHC class II molecules, costimulatory molecules on DCs and induces a highly efficient antigen-specific CTL response in vitro [31] (Figure $1(\mathrm{~b})$ ).

\section{Stimulating the Maturity of Dendritic Cells and Enhancing the Activation of T Cells}

Tumor-antigen-loaded DCs are capable of triggering protective T-cell responses, especially by activating the CTL response in lymphoid organs. However, without a maturation signal (i.e., a stimulatory adjuvant), DCs cannot elicit the desired CTL response. When DCs present antigens in a stable state without an immunogenic maturation signal, regulatory $\mathrm{T}$ cells $\left(\mathrm{T}_{\mathrm{reg}}\right)$ are induced, which both promote tolerance of antitumor responses and directly counters the antitumor response [11]. Thus, stimulatory adjuvant can induce maturation of DCs. What's more, at the same time, stimulatory adjuvant can induce costimulatory molecules (i.e., CD40, CD80, and CD86) on the surface of DCs and the release of cytokines (i.e., IFN- $\alpha$, IFN- $\gamma$, and IL-12) by DCs, which are required for $\mathrm{T}$-cell activation and differentiation (Figure 1(c)).

Many initial attempts at cancer vaccines lacked a consideration of this immune process, specifically the requirement of DCs for a stimulatory adjuvant in order to induce the desired CTLs. Peptide-based vaccines that were used to treat thousands of patients often lacked an effective DC-activating adjuvant [36]. On the one hand, free peptides may be rapidly cleared before being loaded onto DCs because of their poor pharmacokinetic properties, so their half-lives may also be short. On the other hand, without an adjuvant, DCs might remain in the steady state and gradually promote tolerance rather than immunity. As a result, the CTL response to the selected tumor antigens is poor and therapeutic benefit is minimal [37]. Because the function and importance of DCs in stimulating CTL responses is well known now, effective DC-activating adjuvants are increasingly being used in vitro and in vivo. For example, IL-2 is an important stimulatory adjuvant for maintaining DC growth and has received FDA approval for use in melanoma and renal cell cancer. Granulocyte-macrophage colony-stimulating factor (GM-CSF) is necessary for the growth and differentiation of DCs, and tumor-necrosis factor- $\alpha$ (TNF- $\alpha)$ can promote DC maturation and antigen-presenting capabilities [38, 39].

TLR ligands are the ideal adjuvants, allowing a variety of functions required for CTL activation. Firstly, TLR ligands induce the maturation of immature DCs into potent DCs. Secondly, TLR ligands induce the expression of costimulatory molecules (CD40, CD80, and CD86) on DCs that are required for T-cell activation. Thirdly, TLR-induced cytokines, principally IL-12, IFN- $\alpha$, and IFN- $\gamma$, guide T-cell differentiation toward either CD8+ cytotoxic lymphocytes or CD4+ T helper type 1 (Th1) cells [40]. Several TLR ligands have shown significant promise for the treatment of cancer for example, the TLR7 agonist imiquimod that 


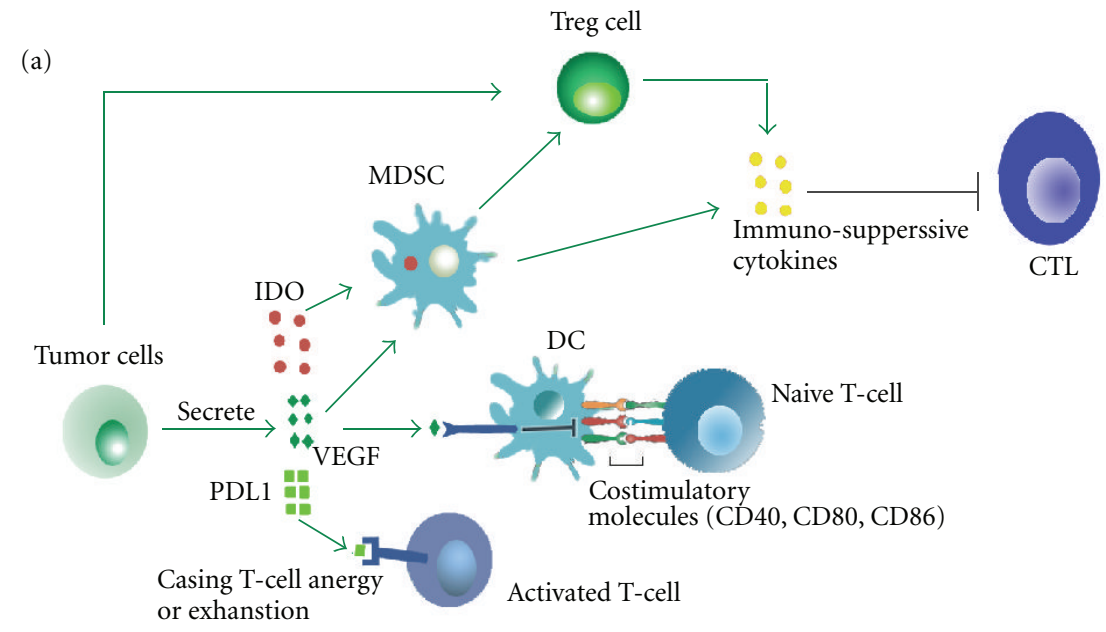

(b)
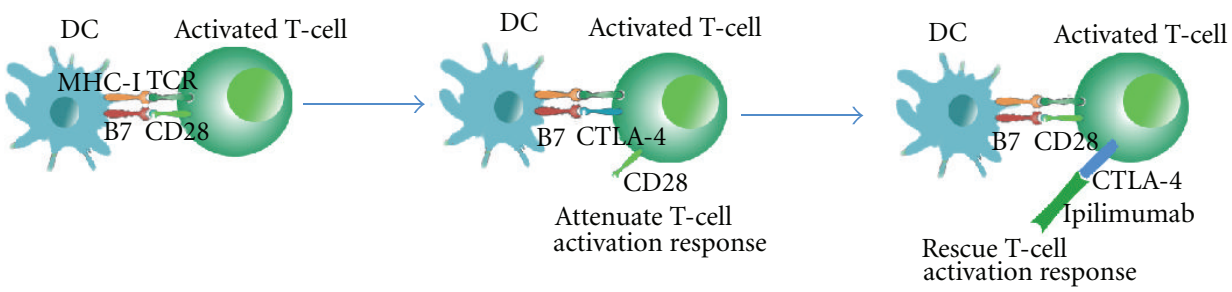

FIgURE 2: The regulation of CTLs in the tumor microenvironment. (a) Tumor-induced immunosuppressive mechanisms in the tumor microenvironment. (b) Activated T cells express more CTLA-4, which competitively engages B7 on the surface of DCs to attenuate T-cell activation. The anti-CTLA-4 antibody ipilimumab rescues the CTLA-4-induced immunosuppression.

is currently approved for treatment of superficial basal cell carcinoma [41]. Although monotherapy with TLR agonists may provide a benefit in certain types of cancer, our interest is centered on the role of TLR agonists as stimulatory adjuvants. Encouraging results have been reported in human melanoma with an antitumor peptide vaccine using CpGODN (a TLR agonist) as an adjuvant, which promotes the generation of rapid and strong CTL antitumor responses [42]. Researchers also confirm that TLR agonists facilitate vaccine-induced CTL responses in many different mouse tumor models [43]. Therefore, its use as a vaccine adjuvant is perhaps the most extensively explored application for TLR agonists [44].

\section{Enhancing the Effect of Vaccine-Induced CTL Antitumor Immune Responses in the Tumor Microenvironment}

After capture, processing and presentation of tumor antigens by DCs and activation of CTLs, activated CTLs must enter the tumor microenvironment to perform their functions; at this point, a variety of negative regulatory signals interact and form a network to resist the immune response. These negative regulatory signals mainly originate from tumor cells [45] and stromal cells such as immune cells, inflammatory cells, endothelial cells, and fibroblasts [46]. Tumor-induced mechanisms of immunosuppression are as follows: (i) tumor cells expansion or enabling the local accumulation of $\mathrm{T}_{\text {reg }}$ cells, which release suppressive cytokines and serve to silence immune responses $[47,48]$, (ii) tumor cells also encourage the formation of myeloid-derived suppressor cells (MDSCs), which use indoleamine 2,3-dioxygenase (IDO) released by tumor cells to silence the responses of cytotoxic CD8+ T cells and helper $\mathrm{CD} 4+\mathrm{T}$ cells while simultaneously promoting the formation of $\mathrm{T}_{\text {reg }}$ cells [49], and (iii) tumor cells secrete a variety of products including the above-mentioned IDO, vascular endothelial growth factor (VEGF) and surface molecules such as PD-L1. VEGF promotes the expansion of MDSCs, blocks the maturation of DCs, and inhibits the expression of costimulatory molecules [50, 51]. PD-L1 engages receptors on the surfaces of activated $\mathrm{T}$ cells and causes T-cell anergy or exhaustion [52, 53] (Figure 2(a)). In addition, $\mathrm{T}$ cells express other receptors that negatively regulate the immune response in the tumor microenvironment. For example, CTLA-4 is a well-studied key negative regulator. When DCs present peptide epitopes to the T-cell receptor (TCR) and simultaneously present $\mathrm{B} 7$ costimulatory molecules (CD80, CD86) to CD28 on specific antitumor T cells, these T cells become activated. CTLA-4 is subsequently upregulated and competitively engages $\mathrm{B} 7$ to attenuate $\mathrm{T}$ cell responses [54] (Figure 2(b)). LAG-3 is another negative receptor on the $\mathrm{T}$ cell surface that not only limits the activity of CD8+ and CD4+ T cells but also augments the activity of $\mathrm{T}_{\text {reg }}$ cells $[55,56]$. Moreover, macrophages polarize to 
type 2 macrophages (M2) because of hypoxia in the tumor microenvironment. M2-like macrophages secrete and release cytokines such as CCL17, CCL22, or CCL24, which recruit $\mathrm{T}_{\text {reg }}$ cells to the local environment [57]. However, there are still a large number of immunosuppressive mechanisms in the tumor microenvironment which are not mentioned in this paper.

Thus, even if pathologically induced auto-CTLs could avoid negative regulatory signals and overcome diverse immunosuppressive networks within the tumor microenvironment, they would still be too weak to promote tumor destruction. Although vaccines (e.g., Sipuleucel-T [58]) can be used to increase the number of CTLs, immunosuppression still occurs in the tumor microenvironment and can attenuate vaccine-induced CTLs. For this reason, a larger number of targeted drugs have been designed and used in clinical trials to counteract negative regulatory mechanisms. Imatinib can decrease IDO expression and lead to an increased ratio of CTLs to $\mathrm{T}_{\text {reg }}$ cells in a mouse model of gastrointestinal stromal tumors [59]. Larmonier et al. reported that imatinib has a direct inhibitory effect on $\mathrm{T}_{\text {reg }}$ cells, including decreased numbers of $\mathrm{T}_{\mathrm{reg}}$ cells and weak immunosuppressive capacity. The combination of imatinib plus a DC vaccine resulted in decreased numbers of $\mathrm{T}_{\text {reg }}$ cells, increased T-cell-derived IFN $\gamma$ production and fewer metastases compared with either therapy alone in a $\mathrm{BCR}^{-} \mathrm{ABL}^{-}$lymphoma model [60]. The targeted drug sunitinib, a multityrosine kinase inhibitor that blocks VEGFR function, decreased the function and number of MDSCs in the tumor microenvironment of a mouse model of colon cancer [49]. Consequently, sunitinib inhibited the number and function of $\mathrm{T}_{\text {reg }}$ cells and augmented the activation of cytotoxic CD8+ $\mathrm{T}$ cells and helper CD4+ $\mathrm{T}$ cells. Furthermore, sunitinib diminished the expression of PD1, PD-L1, and CTLA-4. The combination of a DC vaccine plus sunitinib prolonged survival compared with either agent alone in a B16 melanoma model [61]. The anti-CTLA-4 antibody ipilimumab has recently been approved by the FDA for use as a first-line or second-line therapy in patients with advanced melanoma. Previous studies have confirmed the fundamental importance of CTLA-4 in controlling Tcell function, and CTLA-4 ligation is also important for the immune suppressive function of $\mathrm{T}_{\text {reg }}$ cells $[62,63]$. Hodi et al. reported clinical trial data that led to the FDA approval of anti-CTLA-4 for the treatment of metastatic melanoma. Their data indicates that ipilimumab, most notably, achieves durable benefits for more than 2.5 years [64]. A subsequent clinical trial confirmed that ipilimumab plus standard care improved overall survival compared with standard care alone (11.2 months versus 9.1 months) and significantly increased the proportion of surviving patients for at least 3 years of followup (20.8\% versus $12.2 \%)$ [65].

\section{Overcoming the Resistance of Tumor Cells to CTL-Mediated Cytotoxic Effects}

As the most important function of vaccine-induced CTLs, CTL-mediated cytotoxic effects play a critical role in immunity against cancers. CTLs have the ability to kill tumor cells following the interaction of their TCR with a specific MHC-I complex on tumor cells. This process is mainly mediated by the ability of effector CTLs to release perforin, granzyme, and TNF- $\alpha$, or to express Fas ligand $[66,67]$. Cytotoxicity proceeds through a multistep mechanism: (i) the TCR combines with a specific MHC-I complex on tumor cells, and then the remodeling of the actin cytoskeleton, reorganization of the cell surface, and repositioning of cytoplasmic proteins result in the formation of a so-called immune synapse [68], (ii) polarization of the microtubule organizing center (MTOC) toward the target occurs, and lytic granules move along the microtubules toward the MTOC, and (iii) lysis occurs through the polarized release of the content of cytotoxic granules (a process also referred to as degranulation) or expression of Fas ligand on the CTL surface [69].

The lytic pathway uses mainly two types of mechanisms to kill target cells: one is perforin mediated and another is Fas ligand (FasL) based [66]. The perforin-mediated mechanism is believed to involve secreted perforin, which forms "pores" in the target membrane. Then co-secreted granzymes pass through the "pores," enter cells and activate a cascade of caspases including caspase- 3 and caspase- 8 [70-72]. The FasL-based mechanism involves crosslinking of the cell surface death receptor Fas expressed on target cells induced by cell surface FasL expressed on CTLs. Crosslinked Fas rapidly induces the assembly of an intracellular "death-inducing signaling complex" (DISC), which recruits and activates caspase- 8 and ultimately activates caspase-3, which serves as a common substrate for these two killing mechanisms [72-75]. Both of these mechanisms eventually induce apoptosis of target cells.

Although CTLs have the capacity to efficiently kill tumor cells, tumor cells may develop escape mechanisms to evade CTL-mediated killing. For instance, tumor cells acquire resistance to perforin and granzyme or alter the expression of death receptors. In addition, the genetic instability of tumor cells can lead to the alteration of proapoptotic regulators, one of the most important being p53. It has been demonstrated that the p53 status of tumor cells has a key role in determining the fate of the antitumor CTL response, because it regulates Fas receptor expression, cellular FLICE/caspase8 inhibitory protein (cFLIP) short protein degradation, and CD95-mediated apoptosis [76]. In addition, tumor cells can lower the expression of MHC class I molecules to avoid detection or increase the expression of antiapoptotic proteins in order to resist cytotoxic mechanisms [77].

Therefore, the combination of vaccines and some targeted drugs may counteract these escape mechanisms and improve CTL-mediated tumor destruction. (Figure 3) In contrast to the escape mechanism described above, some targeted drugs enhanced sensitization of tumor cells to CTLmediated destruction [14]. For example, the proteasome inhibitor bortezomib sensitizes tumor cells toward adoptive CTL attack [78]. A preclinical trial of bortezomib plus vaccination with DNA encoding a tumor-specific protein is planned. The combination of an HPV E7-based vaccine and bortezomib prolonged the steady state of the disease, in contrast to either therapy alone, which had minimal 


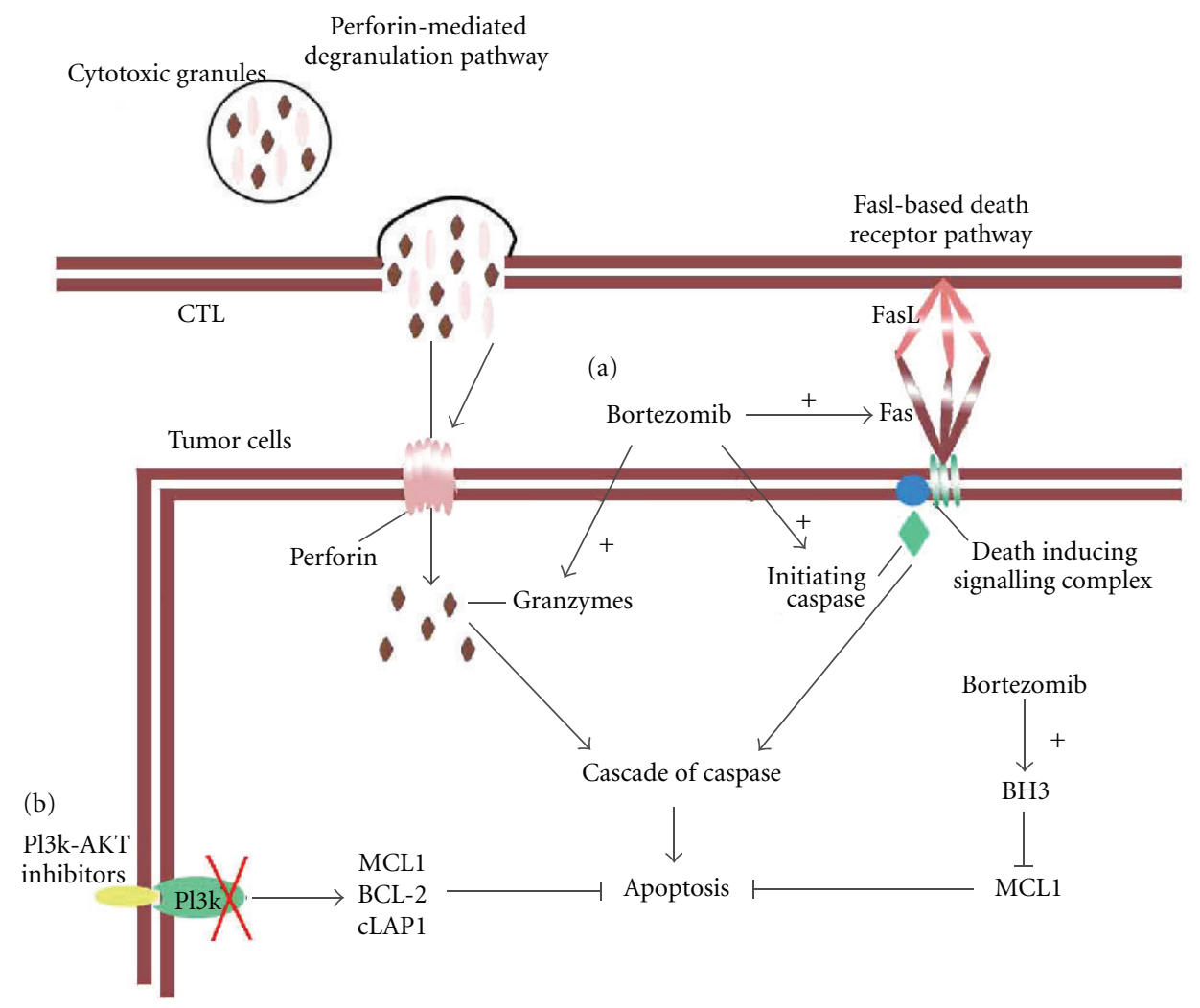

Figure 3: Overcoming the resistance of tumor cells to CTL-mediated cytotoxic effects. (a) Targeted drugs (e.g., bortezomib) sensitize tumor cells toward CTL attack through perforin-mediated and Fas-ligand- (FasL-) based pathways. (b) Targeted drugs (e.g., PI3 K-AKT inhibitors) inhibit antiapoptotic proteins to promote cytotoxic effects.

effects [79]. The increased survival correlated with enhanced sensitivity of the bortezomib-treated tumor cells to killing by E7-specific CTLs. The possible mechanisms are as follows: (i) bortezomib may enhance the baseline activity of granzyme and caspase- 8 in tumor cells, which could provide sensitization towards apoptosis [78], (ii) bortezomib may sensitize tumor cells to CTL-mediated lysis through enhanced expression of NOXA [51], a $\mathrm{BH} 3$ protein that sequesters potent antiapoptotic proteins such as myeloid cell leukemia sequence 1 (MCL1) [80], or (iii) bortezomib may increase tumor cell surface expression of the TNF-related apoptosis-inducing ligand receptor DR5 and Fas, which further augments NK cell cytotoxicity [81]. In addition, some evidence suggests that bortezomib can also sensitize tumor cells to NK cell-mediated lysis by downregulating peptide loading and MHC class I molecule expression. This is due to bortezomib inhibiting the proteasome that generates peptide epitopes for MHC class I molecules, rendering tumor cells more susceptible to killing by NK cells [82].

PI3 K-AKT inhibitors are another class of targeted drugs that sensitize tumor cells to CTL-mediated destruction through PI3 K-AKT pathway inhibition; this eliminates some prosurvival signals such as expression of antiapoptotic proteins, thereby increasing tumor cell lysis by perforin and granzymes released from CTLs [83, 84]. Hähnel et al. confirmed that tumor cells in which AKT can be inducibly expressed showed enhanced resistance to CTL killing upon AKT activation compared with noninduced cells. The enhanced resistance involved an increase in MCL1 expression, which is an antiapoptotic protein [83]. Noh et al. demonstrated that a tumor cell line selected for resistance to vaccination therapy upregulated AKT compared with wildtype cells that are sensitive to vaccination therapy. This result was associated with increased levels of BCL-2, cIAP1, and cIAP2, which are also antiapoptotic proteins. Moreover, Noh et al. demonstrated that inhibiting the upregulation of the AKT pathway increased the CTL-mediated killing of AKTupregulated tumor cells in vitro and AKT inhibition in combination with vaccine-augmented CTL responses [84].

\section{Conclusions}

A variety of strategies have been used to design vaccines that can induce a durable and long-lasting host immune response to fight cancer. However, a series of processes are necessary in order for a vaccine-induced CTL immune response to play a positive role in destroying cancer. In any step in these processes, immune responses may be subject to the regulation of negative signals. Even when CTLs are activated, negative signals causing T-cell anergy or exhaustion may occur in the tumor microenvironment. CTL-mediated cytotoxic effects also are affected by the escape mechanisms of tumor cells. For these reasons, when designing vaccines, we attempt to utilize and regulate DCs to elicit a much 
more potent CTL immune response. This strategy includes antigen-loading techniques in vitro, supplying targeted drugs that facilitate the capture of tumor antigens by DCs in vivo, and using stimulatory adjuvants that promote the whole development and function of DCs. In addition, targeted drugs can be used which block important immunosuppressive signals in the tumor microenvironment, and overcome the tolerance of tumor cells. The strategy of combining vaccines and targeted drugs increases CTL-mediated tumor cell lysis. In the context of advances in the understanding of how immunity, immunosuppression and tolerance regulate antitumor immune responses, immunotherapy and targeted drugs might have complementary and synergistic roles in cancer treatment; therefore, the combination of vaccines and targeted drugs may become a more accepted practice in cancer treatment.

\section{Acknowledgments}

This work was supported by Grants from the Chongqing Science Fund for Distinguished Young Scholars (CSTC, 2009BA5045).

\section{References}

[1] A. Jemal, F. Bray, M. M. Center, J. Ferlay, E. Ward, and D. Forman, "Global cancer statistics," CA Cancer Journal for Clinicians, vol. 61, no. 2, pp. 69-90, 2011.

[2] M. Dougan and G. Dranoff, "Immune therapy for cancer," Annual Review of Immunology, vol. 27, pp. 83-117, 2009.

[3] D. L. Porter, B. L. Levine, M. Kalos, A. Bagg, and C. H. June, "Chimeric antigen receptor-modified T cells in chronic lymphoid leukemia," The New England Journal of Medicine, vol. 365, no. 8, pp. 725-733, 2011.

[4] G. D. Lewis Phillips, G. Li, D. L. Dugger et al., "Targeting HER2-positive breast cancer with trastuzumab-DM1, an antibody-cytotoxic drug conjugate," Cancer Research, vol. 68, no. 22, pp. 9280-9290, 2008.

[5] R. A. Madan and J. L. Gulley, "Sipuleucel-T: harbinger of a new age of therapeutics for prostate cancer," Expert Review of Vaccines, vol. 10, no. 2, pp. 141-150, 2011.

[6] V. Matteo, I. Chiara, H. Ngar-Yee et al., "Strategies for cancer vaccine development," Journal of Biomedicine and Biotechnology, vol. 2010, Article ID 596432, 13 pages, 2010.

[7] I. Mellman and R. M. Steinman, "Dendritic cells: specialized and regulated antigen processing machines," Cell, vol. 106, no. 3, pp. 255-258, 2001.

[8] K. Palucka, J. Banchereau, and I. Mellman, "Designing vaccines based on biology of human dendritic cell subsets," Immunity, vol. 33, no. 4, pp. 464-478, 2010.

[9] L. Zitvogel and G. Kroemer, "Anticancer immunochemotherapy using adjuvants with direct cytotoxic effects," Journal of Clinical Investigation, vol. 119, no. 8, pp. 2127-2130, 2009.

[10] G. G. Kenter, M. J. P. Welters, A. R. P. M. Valentijn et al., "Vaccination against HPV-16 oncoproteins for vulvar intraepithelial neoplasia," The New England Journal of Medicine, vol. 361, no. 19, pp. 1838-1847, 2009.

[11] G. Darrasse-Jèze, S. Deroubaix, H. Mouquet et al., "Feedback control of regulatory $\mathrm{T}$ cell homeostasis by dendritic cells in vivo," Journal of Experimental Medicine, vol. 206, no. 9, pp. 1853-1862, 2009.
[12] A. Jiang, O. Bloom, S. Ono et al., "Disruption of E-cadherinmediated adhesion induces a functionally distinct pathway of dendritic cell maturation," Immunity, vol. 27, no. 4, pp. 610624, 2007.

[13] R. M. Steinman, D. Hawiger, and M. C. Nussenzweig, "Tolerogenic dendritic cells," Annual Review of Immunology, vol. 21, pp. 685-711, 2003.

[14] V. Matthew and D. Glenn, "Combining immunotherapy and targeted therapies in cancer treatment," Nature Reviews Cancer, vol. 12, no. 4, pp. 237-251, 2012.

[15] Y. Nefedova, S. Nagaraj, A. Rosenbauer, C. Muro-Cacho, S. M. Sebti, and D. I. Gabrilovich, "Regulation of dendritic cell differentiation and antitumor immune response in cancer by pharmacologic-selective inhibition of the Janus-activated kinase 2/signal transducers and activators of transcription 3 pathway," Cancer Research, vol. 65, no. 20, pp. 9525-9535, 2005.

[16] Y. Nefedova, P. Cheng, D. Gilkes et al., "Activation of dendritic cells via inhibition of Jak2/STAT3 signaling," Journal of Immunology, vol. 175, no. 7, pp. 4338-4346, 2005.

[17] A. J. Korman, K. S. Peggs, and J. P. Allison, "Checkpoint blockade in cancer immunotherapy," Advances in Immunology, vol. 90, pp. 297-339, 2006.

[18] B. Farsaci, J. P. Higgins, and J. W. Hodge, "Consequence of dose scheduling of sunitinib on host immune response elements and vaccine combination therapy," International Journal of Cancer, vol. 130, no. 8, pp. 1948-1959, 2011.

[19] J. S. Ko, A. H. Zea, B. I. Rini et al., "Sunitinib mediates reversal of myeloid-derived suppressor cell accumulation in renal cell carcinoma patients," Clinical Cancer Research, vol. 15, no. 6, pp. 2148-2157, 2009.

[20] R. D. Schreiber, L. J. Old, and M. J. Smyth, "Cancer immunoediting: integrating immunity's roles in cancer suppression and promotion," Science, vol. 331, no. 6024, pp. 1565-1570, 2011.

[21] K. Palucka, J. Banchereau, and I. Mellman, "Designing vaccines based on biology of human dendritic cell subsets," Immunity, vol. 33, no. 4, pp. 464-478, 2010.

[22] L. Zitvogel and G. Kroemer, "Anticancer immunochemotherapy using adjuvants with direct cytotoxic effects," Journal of Clinical Investigation, vol. 119, no. 8, pp. 2127-2130, 2009.

[23] F. S. Hodi, S. J. O’Day, D. F. McDermott et al., "Improved survival with ipilimumab in patients with metastatic melanoma," The New England Journal of Medicine, vol. 363, no. 8, pp. 711723, 2010.

[24] C. Robert, L. Thomas, I. Bondarenko et al., "Ipilimumab plus dacarbazine for previously untreated metastatic melanoma," The New England Journal of Medicine, vol. 364, no. 26, pp. 2517-2526, 2011.

[25] P. Sharma, K. Wagner, J. D. Wolchok, and J. P. Allison, “Novel cancer immunotherapy agents with survival benefit: recent successes and next steps," Nature Reviews Cancer, vol. 11, no. 11, pp. 805-812, 2011.

[26] C. Palena, S. I. Abrams, J. Schlom, and J. W. Hodge, "Cancer vaccines: preclinical studies and novel strategies," Advances in Cancer Research, vol. 95, pp. 115-145, 2006.

[27] V. Cerundolo, I. F. Hermans, and M. Salio, "Dendritic cells: a journey from laboratory to clinic," Nature Immunology, vol. 5, no. 1, pp. 7-10, 2004.

[28] W. J. Lesterhuis, I. J. M. de Vries, G. J. Adema, and C. J. A. Punt, "Dendritic cell-based vaccines in cancer immunotherapy: an update on clinical and immunological results," Annals of Oncology, vol. 15, no. 4, pp. iv145-iv151, 2004. 
[29] X. M. Tong, S. E. Zheng, A. Bader et al., "Construction of expression vector of HTERT- HIL18 fusion gene and induction of cytotoxic T lymphocyte response against hTERT," European Journal of Medical Research, vol. 13, no. 1, pp. 7-14, 2008.

[30] E. S. Trombetta and I. Mellman, "Cell biology of antigen processing in vitro and in vivo," Annual Review of Immunology, vol. 23, pp. 975-1028, 2005.

[31] P. Correale, C. Botta, M. G. Cusi et al., "Cetuximab \pm chemotherapy enhances dendritic cell-mediated phagocytosis of colon cancer cells and ignites a highly efficient colon cancer antigen-specific cytotoxic T-cell response in vitro," International Journal of Cancer, vol. 130, no. 7, pp. 1577-1589, 2012.

[32] M. E. Wolpoe, E. R. Lutz, A. M. Ercolini et al., "HER2/neu-specific monoclonal antibodies collaborate with HER2/neu-targeted granulocyte macrophage colony-stimulating factor secreting whole cell vaccination to augment CD8+ T cell effector function and tumor-free survival in Her-2/neutransgenic mice," Journal of Immunology, vol. 171, no. 4, pp. 2161-2169, 2003.

[33] A. M. Boruchov, G. Heller, M. C. Veri, E. Bonvini, J. V. Ravetch, and J. W. Young, "Activating and inhibitory IgG Fc receptors on human DCs mediate opposing functions," Journal of Clinical Investigation, vol. 115, no. 10, pp. 2914-2923, 2005.

[34] P. S. Kim, T. D. Armstrong, H. Song et al., "Antibody association with HER-2/neu-targeted vaccine enhances CD8+ T cell responses in mice through Fc-mediated activation of DCs," Journal of Clinical Investigation, vol. 118, no. 5, pp. 1700-1711, 2008.

[35] M. L. Disis, D. R. Wallace, T. A. Gooley et al., "Concurrent trastuzumab and HER2/neu-specific vaccination in patients with metastatic breast cancer," Journal of Clinical Oncology, vol. 27, no. 28, pp. 4685-4692, 2009.

[36] S. A. Rosenberg, J. C. Yang, and N. P. Restifo, "Cancer immunotherapy: moving beyond current vaccines," Nature Medicine, vol. 10, no. 9, pp. 909-915, 2004.

[37] I. Mellman, G. Coukos, and G. Dranoff, "Cancer immunotherapy comes of age," Nature, vol. 480, no. 7378, pp. 480-489, 2011.

[38] M. Carfí, G. Bowe, D. Ferrario, R. Pieters, and L. Gribaldo, "Maintenance and characterization of lymphocytes in human long term bone marrow cultures to study immunotoxicity," Toxicology in Vitro, vol. 24, no. 5, pp. 1395-1403, 2010.

[39] J. A. Klapper, A. A. Thomasian, D. M. Smith et al., "Singlepass, closed-system rapid expansion of lymphocyte cultures for adoptive cell therapy," Journal of Immunological Methods, vol. 345, no. 1-2, pp. 90-99, 2009.

[40] A. Iwasaki and R. Medzhitov, "Toll-like receptor control of the adaptive immune responses," Nature Immunology, vol. 5, no. 10, pp. 987-995, 2004.

[41] Y. C. Chang, V. Madkan, R. Cook-Norris, K. Sra, and S. Tyring, "Current and potential uses of imiquimod," Southern Medical Journal, vol. 98, no. 9, pp. 914-920, 2005.

[42] D. E. Speiser, D. Liénard, N. Rufer et al., "Rapid and strong human $\mathrm{CD}^{+} \mathrm{T}$ cell responses to vaccination with peptide, IFA, and CpG oligodeoxynucleotide 7909," Journal of Clinical Investigation, vol. 115, no. 3, pp. 739-746, 2005.

[43] A. M. Krieg, "Antitumor applications of stimulating Toll-like receptor 9 with CpG oligodeoxynucleotides," Current Oncology Reports, vol. 6, no. 2, pp. 88-95, 2004.

[44] A. M. Krieg, "Therapeutic potential of toll-like receptor 9 activation," Nature Reviews Drug Discovery, vol. 5, no. 6, pp. 471-484, 2006.
[45] G. A. Rabinovich, D. Gabrilovich, and E. M. Sotomayor, "Immunosuppressive strategies that are mediated by tumor cells," Annual Review of Immunology, vol. 25, pp. 267-296, 2007.

[46] N. A. Bhowmick, E. G. Neilson, and H. L. Moses, "Stromal fibroblasts in cancer initiation and progression," Nature, vol. 432, no. 7015, pp. 332-337, 2004.

[47] T. J. Curiel, G. Coukos, L. Zou et al., "Specific recruitment of regulatory $\mathrm{T}$ cells in ovarian carcinoma fosters immune privilege and predicts reduced survival," Nature Medicine, vol. 10, no. 9, pp. 942-949, 2004.

[48] K. Kono, H. Kawaida, A. Takahashi et al., "CD4(+)CD25 $5^{\text {high }}$ regulatory $\mathrm{T}$ cells increase with tumor stage in patients with gastric and esophageal cancers," Cancer Immunology, Immunotherapy, vol. 55, no. 9, pp. 1064-1071, 2006.

[49] J. Ozao-Choy, M. Ge, J. Kao et al., "The novel role of tyrosine kinase inhibitor in the reversal of immune suppression and modulation of tumor microenvironment for immune-based cancer therapies," Cancer Research, vol. 69, no. 6, pp. 25142522, 2009.

[50] C. Alfaro, N. Suarez, A. Gonzalez et al., "Influence of bevacizumab, sunitinib and sorafenib as single agents or in combination on the inhibitory effects of VEGF on human dendritic cell differentiation from monocytes," British Journal of Cancer, vol. 100, no. 7, pp. 1111-1119, 2009.

[51] D. H. Yang, J. S. Park, C. J. Jin et al., "The dysfunction and abnormal signaling pathway of dendritic cells loaded by tumor antigen can be overcome by neutralizing VEGF in multiple myeloma," Leukemia Research, vol. 33, no. 5, pp. 665-670, 2009.

[52] J. Hamanishi, M. Mandai, M. Iwasaki et al., "Programmed cell death 1 ligand 1 and tumor-infiltrating $\mathrm{CD}^{+} \mathrm{T}$ lymphocytes are prognostic factors of human ovarian cancer," Proceedings of the National Academy of Sciences of the United States of America, vol. 104, no. 9, pp. 3360-3365, 2007.

[53] M. E. Keir, M. J. Butte, G. J. Freeman, and A. H. Sharpe, "PD-1 and its ligands in tolerance and immunity," Annual Review of Immunology, vol. 26, pp. 677-704, 2008.

[54] C. A. Chambers, M. S. Kuhns, J. G. Egen, and J. P. Allison, "CTLA-4-mediated inhibition in regulation of $\mathrm{T}$ cell responses: mechanisms and manipulation in tumor immunotherapy," Annual Review of Immunology, vol. 19, pp. 565594, 2001.

[55] C. J. Workman and D. A. A. Vignali, "Negative regulation of T cell homeostasis by lymphocyte activation gene-3 (CD223)," Journal of Immunology, vol. 174, no. 2, pp. 688-695, 2005.

[56] C. T. Huang, C. J. Workman, D. Files et al., "Role of LAG-3in regulatoryT cells," Immunity, vol. 21, no. 4, pp. 503-513, 2004.

[57] A. Facciabene, X. Peng, I. S. Hagemann et al., "Tumour hypoxia promotes tolerance and angiogenesis via CCL28 and T reg cells," Nature, vol. 475, no. 7355, pp. 226-230, 2011.

[58] P. W. Kantoff, C. S. Higano, N. D. Shore et al., "Sipuleucel-T immunotherapy for castration-resistant prostate cancer," The New England Journal of Medicine, vol. 363, no. 5, pp. 411-422, 2010.

[59] V. P. Balachandran, M. J. Cavnar, S. Zeng et al., "Imatinib potentiates antitumor $\mathrm{T}$ cell responses in gastrointestinal stromal tumor through the inhibition of Ido," Nature Medicine, vol. 17, no. 9, pp. 1094-1100, 2011.

[60] N. Larmonier, N. Janikashvili, C. J. Lacasse et al., "Imatinib mesylate inhibits $\mathrm{CD} 4{ }^{+} \mathrm{CD} 25^{+}$regulatory $\mathrm{T}$ cell activity and enhances active immunotherapy against BCR-ABL- tumors," Journal of Immunology, vol. 181, no. 10, pp. 6955-6963, 2008.

[61] A. Bose, J. L. Taylor, S. Alber et al., "Sunitinib facilitates the activation and recruitment of therapeutic anti-tumor 
immunity in concert with specific vaccination," International Journal of Cancer, vol. 129, no. 9, pp. 2158-2170, 2011.

[62] E. A. Tivol, F. Borriello, A. N. Schweitzer, W. P. Lynch, J. A. Bluestone, and A. H. Sharpe, "Loss of CTLA-4 leads to massive lymphoproliferation and fatal multiorgan tissue destruction, revealing a critical negative regulatory role of CTLA-4," Immunity, vol. 3, no. 5, pp. 541-547, 1995.

[63] K. Wing, Y. Onishi, P. Prieto-Martin et al., "CTLA-4 control over Foxp $3^{+}$regulatory T cell function,” Science, vol. 322, no. 5899, pp. 271-275, 2008.

[64] F. S. Hodi, S. J. O’Day, D. F. McDermott et al., "Improved survival with ipilimumab in patients with metastatic melanoma," The New England Journal of Medicine, vol. 363, no. 8, pp. 711$723,2010$.

[65] C. Robert, L. Thomas, I. Bondarenko et al., "Ipilimumab plus dacarbazine for previously untreated metastatic melanoma," The New England Journal of Medicine, vol. 364, no. 26, pp. 2517-2526, 2011.

[66] D. Kagi, F. Vignaux, B. Ledermann et al., "Fas and perforin pathways as major mechanisms of T cell-mediated cytotoxicity," Science, vol. 265, no. 5171, pp. 528-530, 1994.

[67] H. Kojima, N. Shinohara, S. Hanaoka et al., "Two distinct pathways of specific killing revealed by perforin mutant cytotoxic T lymphocytes," Immunity, vol. 1, no. 5, pp. 357364, 1994.

[68] J. C. Stinchcombe, G. Bossi, S. Booth, and G. M. Griffiths, "The immunological synapse of CTL contains a secretory domain and membrane bridges," Immunity, vol. 15, no. 5, pp. 751-761, 2001.

[69] J. C. Stinchcombe and G. M. Griffiths, "Secretory mechanisms in cell-mediated cytotoxicity," Annual Review of Cell and Developmental Biology, vol. 23, pp. 495-517, 2007.

[70] S. P. Cullen and S. J. Martin, "Mechanisms of granuledependent killing," Cell Death and Differentiation, vol. 15, no. 2, pp. 251-262, 2008.

[71] J. Pardo, R. Wallich, P. Martin et al., "Granzyme B-induced cell death exerted by ex vivo CTL: discriminating requirements for cell death and some of its signs," Cell Death and Differentiation, vol. 15 , no. 3, pp. 567-579, 2008.

[72] D. Hassin, O. G. Garber, A. Meiraz, Y. S. Schiffenbauer, and G. Berke, "Cytotoxic T lymphocyte perforin and Fas ligand working in concert even when Fas ligand lytic action is still not detectable," Immunology, vol. 133, no. 2, pp. 190-196, 2011.

[73] F. C. Kischkel, S. Hellbardt, I. Behrmann et al., "Cytotoxicitydependent APO-1 (Fas/CD95)-associated proteins form a death-inducing signaling complex (DISC) with the receptor," EMBO Journal, vol. 14, no. 22, pp. 5579-5588, 1995.

[74] T. B. Kang, G. S. Oh, E. Scandella et al., "Mutation of a selfprocessing site in caspase- 8 compromises its apoptotic but not its nonapoptotic functions in bacterial artificial chromosometransgenic mice," Journal of Immunology, vol. 181, no. 4, pp. 2522-2532, 2008.

[75] A. Strasser, P. J. Jost, and S. Nagata, "The many roles of FAS receptor signaling in the immune system," Immunity, vol. 30, no. 2, pp. 180-192, 2009.

[76] A. Hamaï, H. Benlalam, F. Meslin et al., "Immune surveillance of human cancer: if the cytotoxic T-lymphocytes play the music, does the tumoral system call the tune?" Tissue Antigens, vol. 75 , no. 1, pp. 1-8, 2010.

[77] F. M. Marincola, E. M. Jaffee, D. J. Hickljn, and S. Ferrone, "Escape of human solid tumors from t-cell recognition: molecular mechanisms and functional significance," Advances in Immunology, no. 74, pp. 181-273, 2000.
[78] J. M. Seeger, P. Schmidt, K. Brinkmann et al., "The proteasome inhibitor bortezomib sensitizes melanoma cells toward adoptive CTL attack," Cancer Research, vol. 70, no. 5, pp. 18251834, 2010.

[79] C. W. Tseng, A. Monie, C. Y. Wu et al., "Treatment with proteasome inhibitor bortezomib enhances antigen-specific $\mathrm{CD}^{+} \mathrm{T}$-cell-mediated antitumor immunity induced by DNA vaccination," Journal of Molecular Medicine, vol. 86, no. 8, pp. 899-908, 2008.

[80] L. Chen, S. N. Willis, A. Wei et al., "Differential targeting of prosurvival Bcl-2 proteins by their $\mathrm{BH} 3$-only ligands allows complementary apoptotic function," Molecular Cell, vol. 17, no. 3, pp. 393-403, 2005.

[81] W. H. D. Hallett, E. Ames, M. Motarjemi et al., "Sensitization of tumor cells to NK cell-mediated killing by proteasome inhibition," Journal of Immunology, vol. 180, no. 1, pp. 163$170,2008$.

[82] J. Shi, G. J. Tricot, T. K. Garg et al., "Bortezomib downregulates the cell-surface expression of HLA class I and enhances natural killer cell-mediated lysis of myeloma," Blood, vol. 111, no. 3, pp. 1309-1317, 2008.

[83] P. S. Hähnel, S. Thaler, E. Antunes, C. Huber, M. Theobald, and M. Schuler, "Targeting AKT signaling sensitizes cancer to cellular immunotherapy," Cancer Research, vol. 68, no. 10, pp. 3899-3906, 2008.

[84] K. H. Noh, T. H. Kang, J. H. Kim et al., "Activation of Akt as a mechanism for tumor immune evasion," Molecular Therapy, vol. 17, no. 3, pp. 439-447, 2009. 


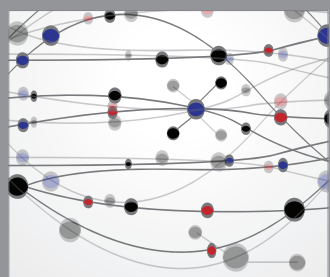

The Scientific World Journal
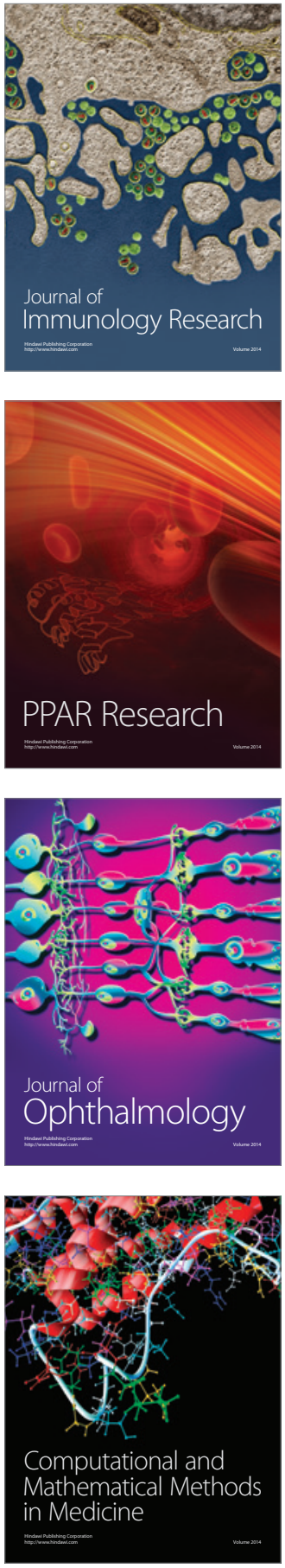

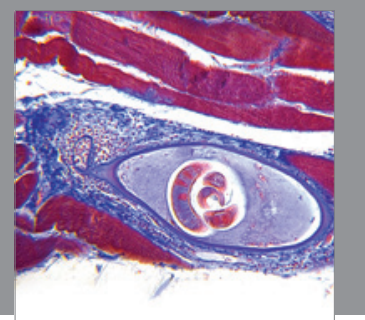

Gastroenterology

Research and Practice
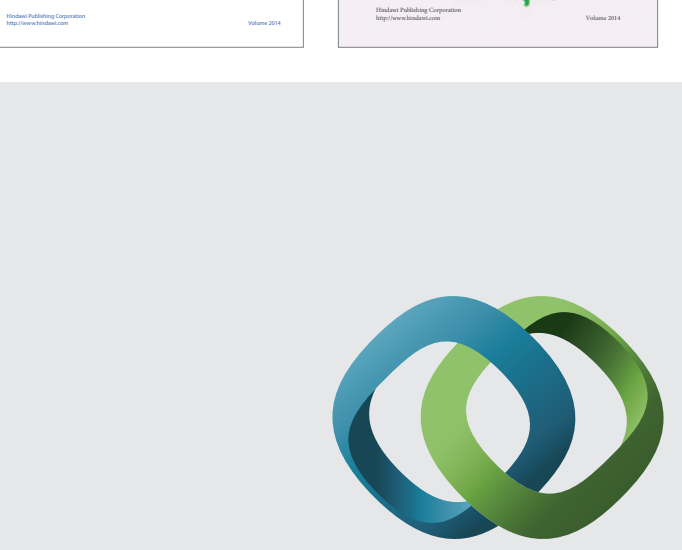

\section{Hindawi}

Submit your manuscripts at

http://www.hindawi.com
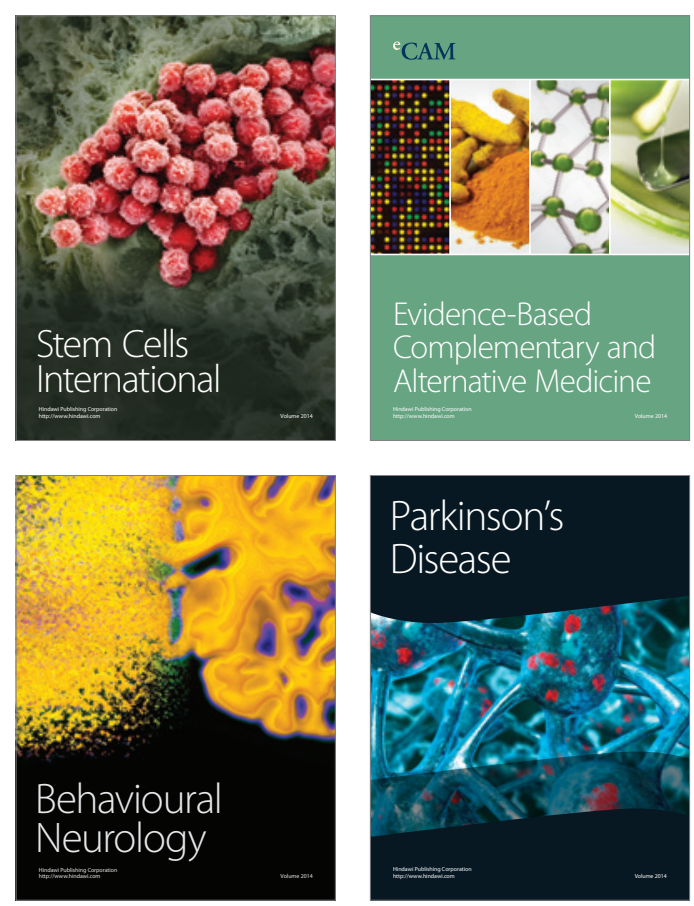

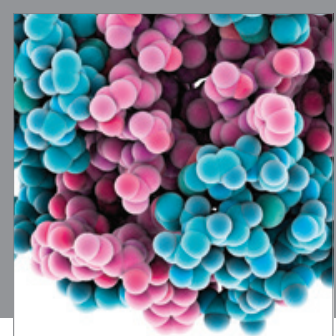

Journal of
Diabetes Research

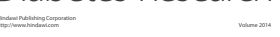

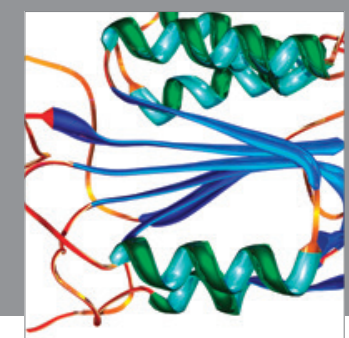

Disease Markers
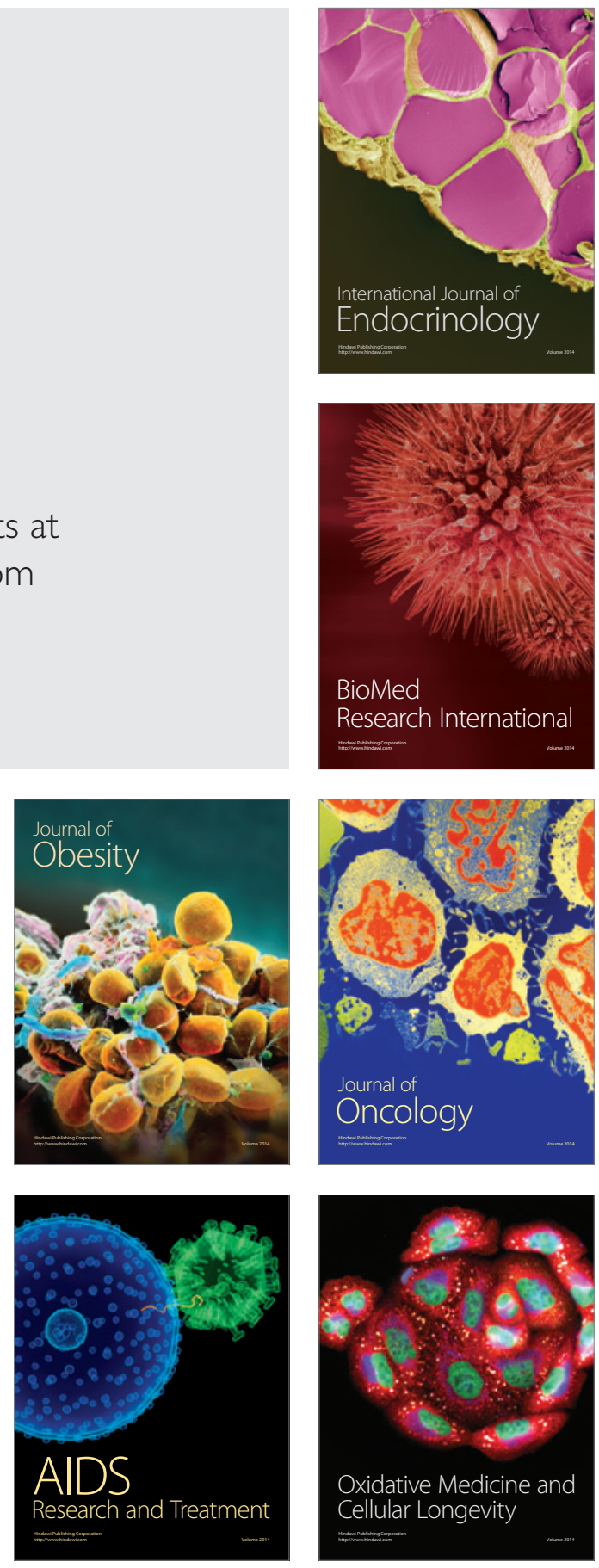